\title{
Research on Sensorless Control Strategy of Motor Controller for Electric Bicycle
}

\author{
Tengfei Lyu ${ }^{1, a^{*}}$, Changchun $\mathrm{Chi}^{2, \mathrm{~b}}$ and Yaofei $\mathrm{Yu}^{3, \mathrm{c}}$ \\ ${ }^{1}$ Shanghai Dianji University, Shanghai, China \\ ${ }^{2}$ Shanghai Dianji University, Shanghai, China \\ ${ }^{3}$ Shanghai Dianji University, Shanghai, China \\ a269621493@qq.com, b chicc@sdju.edu.cn, ${ }^{\text {c3 } 342150148 @ q q . c o m ~}$
}

Keywords: electric bicycle, motor controller, sensorless control

Abstract. This paper research on sensorless control of brushless dc motor control for electric bicycle. Based on the work requirements of electric bicycles, this paper introduces some method to improve the start of the motor and the detection of the motor's rotor position, expand the speed range of electric bicycle without hall and solve some problem in starting process. Finally, this paper verifies the feasibility of the scheme on the electric bicycle.

\section{Introduction}

Now most of the control strategy of electric bicycle give priority to sensor control strategy. Sensorless control strategy is complementary. When the hall sensors can work normally in the electric motor, the sensor control strategy will be adopted. When the hall sensors are broken, the controller will switch into the sensorless control strategy, in order to ensure the electric bicycle can still run without hall sensor [1-2]. Although sensorless control strategy can ensure electric bicycle working, it can't satisfy customers' demand for high quality of running on electric bicycle [3-4]. Compared with the sensor control strategy, the sensorless control strategy of electric bicycle are still many deficiencies.

Those are:

1) The motor speed, can be adjusted, is low.

2) There are the dithering phenomenon in starting process.

\section{The sensorless control method}

The rotor detected method of sensorless control. Based on the electric bicycle's operation environment and operation requirements of the motor, this paper improves the back EMF $\mathrm{ZCP}$ (electromagnetic force zero-crossing point), optimizes the voltage comparison circuit, and enlarges the speed range of the method.

In the process of normal operation of the simplified equation of brushless dc motor can be written as follows [5-6]:

$$
\left[\begin{array}{l}
U_{a} \\
U_{b} \\
U_{\sigma}
\end{array}\right]=\left[\begin{array}{ccc}
R & 0 & 0 \\
0 & R & 0 \\
0 & 0 & R
\end{array}\right]\left[\begin{array}{c}
i_{a} \\
i_{b} \\
i_{a}
\end{array}\right]+\mathbf{P}\left[\begin{array}{ccc}
L-M & 0 & 0 \\
0 & L-M & 0 \\
0 & 0 & L-M
\end{array}\right]\left[\begin{array}{l}
i_{a} \\
i_{b} \\
i_{a}
\end{array}\right]+\left[\begin{array}{l}
e_{a} \\
e_{b} \\
e_{\sigma}
\end{array}\right]
$$

(In the Eq. $1, U_{a}, U_{b}, U_{c}$ are the three-phase voltages of brushless dc motor, $t_{a}, t_{b}, t_{c}$ are the three phase currents, $e_{a}, e_{b}, e_{c}$ are the three phase opposite potentials, $\mathrm{R}$ is the resistance of three-phase, $\mathrm{M}$ is the mutual inductance between the phase winding, $\mathrm{L}$ is the self-inductance of the three-phase winding, and $\mathrm{P}$ is the differential operator $d / d t$ )

We can see that[4] :

$$
U_{x}=e_{x}+U_{n}
$$


In the Eq. 2, x represents the no conduction phase, and $U_{n}$ represents the neutral point voltage [7]. From the above equation, we can see that the no conduction phase winding terminal voltage and the neutral point voltage can be compared through hardware circuit[8] to get the output of the comparator, and it is the brushless dc motor of the back EMF zero signal[9].

The back EMF ZCP introduces the low-pass filter [10]. It needs to be established table according to the phase shift Angle under different rotational speed form to query the compensating phase shift Angle, which can make the rotor position detection inaccurate[11]. When the motor speed is very low or static, it is difficult to accurately detect the zero signal. It limits the motor's adjustable speed range [12].

To enlarge the adjustable speed range of electric bicycle, the partial pressure resistance of the back EMF ZCP to be replaced by a pair of countered diode. Through reverse phase cut-off and positive phase conduction principle of the diode can be used to accurately detect the back EMF zero. Its hardware detection principle block diagram is shown in Figure 1.

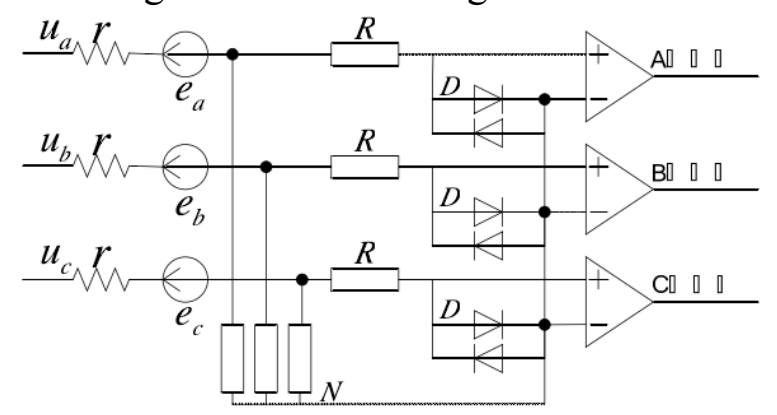

Figure 1 the improved "back EMF method" detection circuit principle diagram

The motor starting method. The starting technology is very important to the performance of the electric bicycle. In order to reduce the dithering of sensorless electric bicycle in the process of starting. This paper introduces the inductive method into the three-step startup method. The closed loop starting method based on the inductive method is used to starting the electric bicycle. The starting mothed can be divided into the following three processes:

The finding of rotor's initial position: the rotor's initial position can be found by comparing the current value of the stator inductance, when the stator are applied several testing voltage vectors [13]. This method can avoid the reversal phenomenon in the process of rotor's position found.

Closed loop accelerating: six accelerating voltage vectors are applied, in turn, to the motor. And two consecutive short time voltage vector are applied to the motor in the interval of the each two of the six accelerating voltage vectors in order to judge whether the rotor is in the range of expected. The next set of voltage vector is decided by the current rotor position. so the acceleration process is kept in the closed loop state. So the dithering phenomenon in the accelerate process can be avoided.

The process of synchronous operation: when meet the switching conditions that the controller can continuous detect the position of the rotor, the control method switch to the sensorless position controlling. Or the motor will be kept in starting process until the switching conditions met.

\section{Introduction of sensorless control system}

The improve of hardware system. This paper design the motor control system of electric bicycle with 32-bit STM MCU as the core, including power supply circuit, the handlebar signal detection circuit, three-phase full bridge inverter circuit, inverter drive circuit, the back EMF ZCP circuit, current detection circuit, over current protection circuit, display and alarm circuit. As shown in Figure 2. 


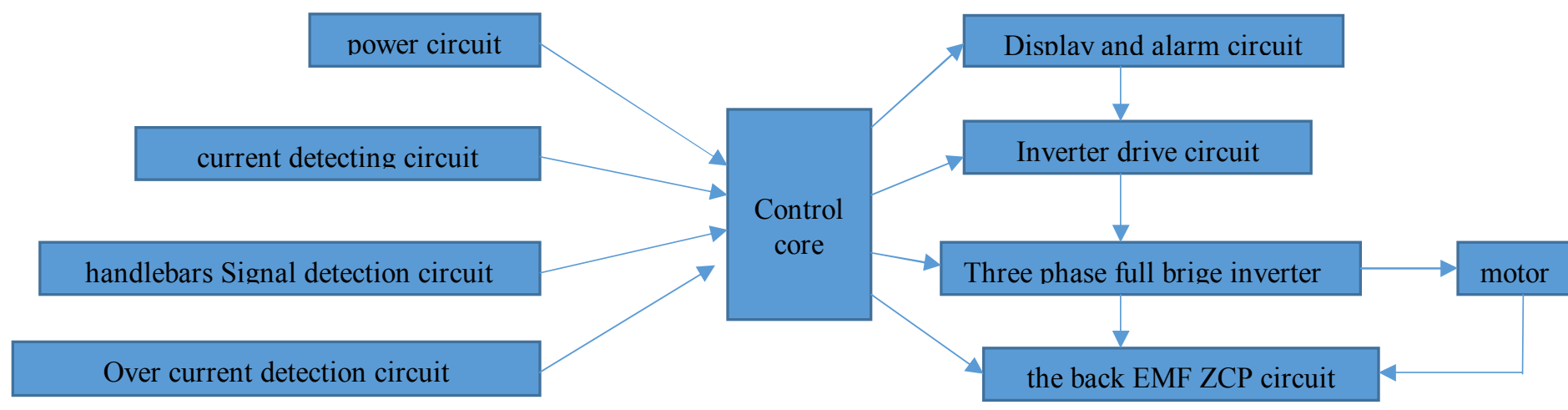

Figure 2 the block diagram of sensorless control of brushless dc motor

In the improved sensorless rotor position detection circuit, when the back EMF in the brushless dc motor is positive half-wave, the noninverting input voltage of comparator is a positive voltage of a diode, and the comparator output high level. When the back EMF in the brushless dc motor is negative half-wave, the noninverting input voltage of comparator is a negative voltage of a diode, and the comparator output low level. As a result, the comparator output a series of square waves with duty ratio $50 \%$ in theory, and the back EMF ZCP are each jump of the square wave signal.

As a result, the noninverting input voltage of comparator is equal to the voltage of a diode. That avoided the inaccurate accuracy because of the potential voltage change. That can solve the problem of detecting position in the case of low speed and small counter potential. This method can be accurately realize the function of the position detection under the low speed, so it can achieve the function of a wide range of speed regulation.

The improvement of software system. On the top of the program, the software consists of Initialize program and the main program. The main program is launched after completion of the necessary initialization. The body of the software system is an infinite loop, continually determines operational status, and switches between three different states. Only when the system appear over current protection, under-voltage protection or hardware failure, the main program is out the infinite loop and to the end. Then, the system will restart [14].

In the improved hardware circuit, antiparallel diode pair are connect to the back EMF zero detection circuit in series. The diode voltage drop causes the phase shift of the back EMF zero point. The electrical angle of the phase shift is found fixed in the experiment. So the proper phase compensation is necessary in programming to get the best commutation signals. The flow chart of rotor position detection of sensorless control is shown in Figure 3.

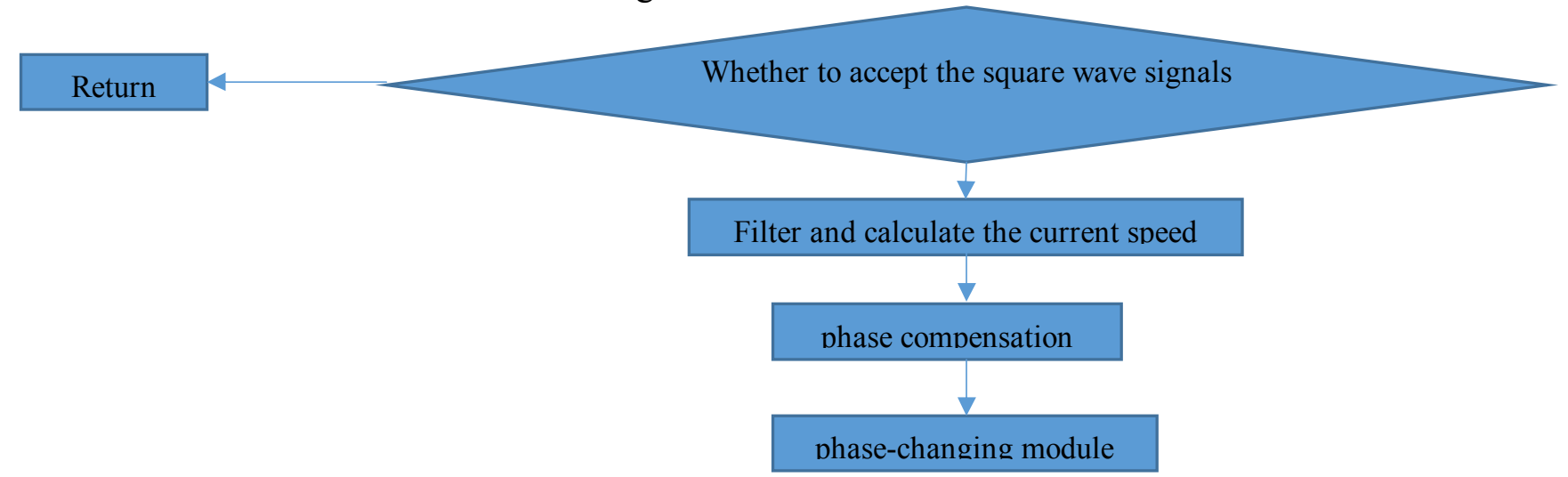

Figure. 3 the flow chart of rotor position detection of sensorless control

\section{Experiment conclusion}

In this paper, we design the sensorless detection circuit, and transplant it to the motor controller S91-T5, and remove the original hall detection circuit on the controller. And then, through the experiment, this paper proves that the sensorless drive strategy is feasible. 
The proposed theory is verified on the experiment platform of the improved S91-T5 controller and a $60 \mathrm{~V} / 30 \mathrm{~A}$ motor.

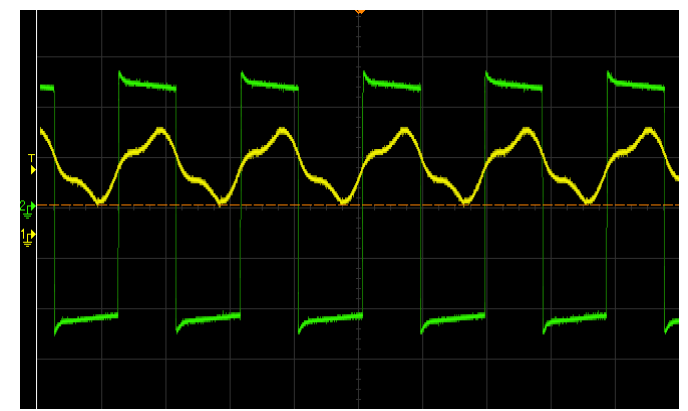

Figure 4 input waveform and output waveform of sensorless detection circuit

As shown in Figure 4, the yellow wave is one of motor stator's three phase voltage waveform after filtered in position sensorless detection circuit. The green wave is the comparator output wave which is the result of the yellow waveforms comparing with the analog neutral point. The output represents the waveform of rotor position, namely the output waveform of the sensorless detection circuit.

The Figure 4 shows that the back EMF zero signal in A phase captured by circuit accurately and timely, the positive and negative conversion of the output waveform appear in the counter electromotive force zero point, so the position of the rotor were determined accurately.

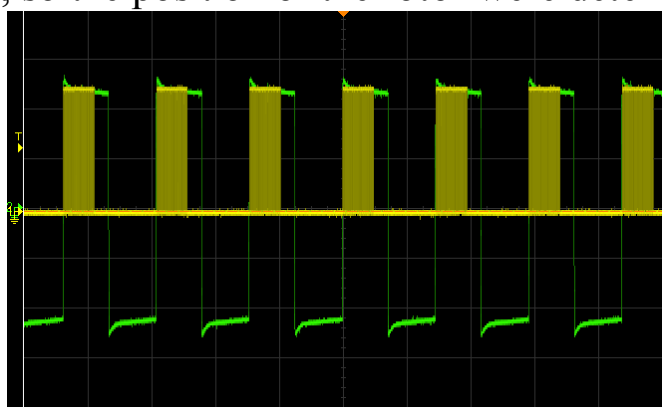

Figure 5 the output waveform of sensorless detection circuit and the driving waveforms of the MOS pipe on the up bridge arm

As shown in Figure 5, the green wave is the output waveform of sensorless detection circuit whose input is the A phase voltage. the waveform can reflect the position of the rotor. Yellow waveform is on behalf of the driving waveform of the A phase circuit. It controls the MOS tubes of A phase circuit turning on and off.

The Figure 5 shows that through processed by the STM MCU detect, the waveform of rotor position becomes corresponding PWM waveform to drive MOS tube of the corresponding phase.

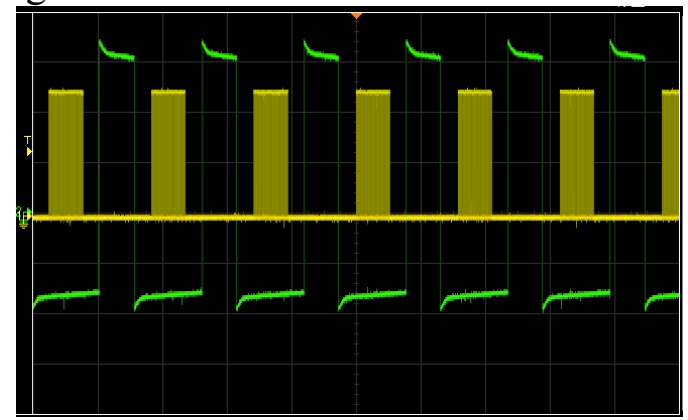

Figure 6 the driving waveforms of the MOS pipe on the up and down bridge arm

As shown in Figure 6, yellow wave is the driving waveform of the MOS pipe on the up bridge arm. Green wave is the driving waveform of the MOS pipe on the down bridge arm.

The Figure 6 shows that basing on the output waveform of sensorless rotor position detection circuit, STM MCU output corresponding driving waveform of MOS tube. The experiment has proved that the driving waveform completely satisfying the requirement of the brushless dc motor driving. So the sensorless controlling strategy of motor controller for electric bicycle in this paper is rational. 


\section{Conclusion}

Based on the work requirements of small brushless dc motor on electric bicycle, this paper improves the traditional counter electromotive force passing zero method to enlarge the speed range of BLDCM and simplify the phase compensation. Based on inductive method, this paper introduce the rotor position closed loop starting method of BLDCM to avoid the phenomenon of the reversal of the motor rotor when fixing position. Starting and accelerating stage with closed loop acceleration method instead of the open loop acceleration method, the electric bicycle is insusceptible to the changing load torque. So it is not easy to step out and it meets the requirements of the running environment of electric bicycle motor. And the feasibility of the proposed scheme is verified by experiment in this paper.

\section{References}

[1] Wenchen Xu, Research on adaptive charging device of electric bicycle [M]. 2013

[2] Xianquan Li, Chunling Yang, The design of the new sensorless BLDCM control systemfor electric vehicle [J]. Electronic Technology Application, 2009, (6): 30-33.

[3] Danyun Lin, Wenguang Luo, Hao ZHANG, Ying Cao, Design of controller of brushless DC motor for electric vehicle [J]. Journal of Guangxi Institute of Technology, 2012, (2): 37-40, 50.

[4] Zhichun Wang, Jiqing Song, Jianguo Sun, Permanent Magnet Brushless DC Motor Without Position Sensor Control [J]. Journal of Guangxi Institute of Technology, 2014, (3): 63-65, 68.

[5] Mingji Liu, Yu Yao, Jibin Zou, Zicai Wang, Effect of the Armature Reaction on the Phase of the Commutation E.M.F.in Brushless DC Motors [J]. Micro Motor, 2001, (4): 3-5.

[6] Fuhong Dong, Yanxia Sheng, Zhicheng Ji, Review on Position Estimation Methods of Brushless DC Motor Sensorless Control [J]. Micro Motor, 2003, (5): 39-46.

[7] Jung, S., Kim, Y., Jae, J., \& Kim, J. Commutation Control for Low Commutation Torque Ripple in Position Sensorless Drive of Low-Voltage Brushless DC Motor[J].

[8] Yong Zhao, Research compare on sensorless Control for Brushless DC Motors [D]. Hefei University of Technology, 2012.

[9] Junhe Zhang, Guohe Shu, Haibin Chen, An Analysis of Step-by-step Startup of Brushless DC Motors Based on Back-EMF Inspection [J]. Electrical Automation, 2014, (5): 1-3.

[10] Zhang, X. Z., \& Wang, Y. N. (2011). A novel position-sensorless control method for brushless DC motors. Energy Conversion and Management[J], 52(3), 1669-1676.

[11] Guangwei Meng, Huaishu Li, Hao Xiong, Principle and Research on Start-up Control of Sensorless BLDCM [J]. Explosion-proof motor, 2010, (2): 20-22, 36.

[12] Xiaowei Zhong, Zhecun Song, Gang Xun, Brushless DC Motor of Electric Bicycle Control System Design [J]. Motor and Control Applications, 2011, (1): 20-24.

[13] Jianbo Cao, Binggang Cao, Bin Wang, Position-Sensorless Control for Brushless DC Motor of Electric Vehicle [J]. Journal of Xi'an Jiaotong University, 2008, (5): 597-601.

[14] Xu Yang, Wenlong Zai, A rotor position detection method for sensorless brushlessDC motors used in electric bicycles [J]. New Technology of Electrical Power, 2009, (2): 63-67.

\section{Acknowledgements}

Sponsored by Natural Science Foundation of Shanghai (No.:12zr1411700)

Science and Technology Project of Minhang (No.:2014M H134) 